\section{Cureus}

\title{
Diethylstilbestrol in the Treatment of Castration-resistant Prostate Cancer: A Lower-middle-income Country Experience
}

Azfar Ali ${ }^{1}$, Muhammad Arshad Irshad Khalil ${ }^{1}$, Nouman Khan ${ }^{1}$, Muhammad Abu Bakar ${ }^{2}$, Awais Amjad ${ }^{3}$, Irfan Ahmed ${ }^{4}$, Khurram Mir ${ }^{1}$

1. Surgical Oncology, Shaukat Khanum Memorial Cancer Hospital and Research Center, Lahore, PAK 2. Biostatistics and Epidemiology, Shaukat Khanum Memorial Cancer Hospital and Research Center, Lahore, PAK 3. Surgery, Shaukat Khanum Memorial Cancer Hospital and Research Center, Lahore, PAK 4. Urology, Shaukat Khanum Memorial Cancer Hospital and Research Center, Lahore, PAK

$\square$ Corresponding author: Azfar Ali, skmdrazfarali@gmail.com

Disclosures can be found in Additional Information at the end of the article

\section{Abstract}

Introduction: Prostate cancer is the second most common cancer and the fifth leading cause of death worldwide. Its metastatic stage is associated with considerable morbidity and may lead to death. In Pakistan, given the high levels of economic constraint, patients with castrationresistant metastatic prostate cancer can be treated with cost-effective medications like diethylstilbestrol (DES).

Objectives: The goal of this study was to assess the efficacy and adverse effects of DES when used in patients with castration-resistant prostate cancer (CRPC).

Materials and methods: From January 2011 to December 2016, all medical records of patients with a diagnosis of prostate cancer resistant to the effects of castration presenting at Shaukat Khanum Cancer Hospital and Research Centre, Lahore, were reviewed. All patients were treated with DES (2.5 mg) initially, but the dose was increased for some patients to $5 \mathrm{mg}$ in combination with aspirin $(75 \mathrm{mg})$. The patients were followed clinically with prostate-specific antigen (PSA) value assessment. The PSA response to treatment, time to disease progression, and adverse events were recorded and analyzed using IBM SPSS Statistics for Windows, Version 20.0 (IBM Corp., Armonk, NY).

Results: A total of 91 patients were included in the study, and the mean patient age was $66 \pm 8$ years. The median baseline PSA was $150 \mathrm{ng} / \mathrm{mL}$ (range: $56-626 \mathrm{ng} / \mathrm{mL}$ ), and the median Gleason's score was eight. A total of $90.1 \%$ of patients had metastatic disease at the time of diagnosis. Hormonal ablation was provided with bilateral orchiectomy for 71 patients (78.0\%), and luteinizing hormone-releasing hormone (LHRH) analog was provided for 20 patients (22.0\%). With this treatment, the median time to PSA progression was 597 days. After DES treatment was started, 78 patients $(87.7 \%)$ showed a PSA response, and median time to progression was 212 days. In 24 patients (26.4\%), the PSA response was maintained for more than a year. The PSA response was quantified as a good response (i.e., $\geqslant 50 \%$ PSA drop) or as a partial response (i.e., <50\% PSA drop). The good PSA response was observed in 56 patients (61.5\%) with a median time to progression of 273 days, and 22 patients (24.2\%) had a partial response maintained for 109 days. Thirteen patients (14.3\%) did not respond to DES treatment. The median percent change in PSA was $-55.52 \%$ (range: -99.9 to +422 ). Thromboembolic complication was observed in eight patients (8.7\%) patients while two patients suffered from liver toxicity.

\section{How to cite this article}

Ali A, Khalil M, Khan N, et al. (April 16, 2019) Diethylstilbestrol in the Treatment of Castration-resistant Prostate Cancer: A Lower-middle-income Country Experience. Cureus 11(4): e4470. DOI 10.7759 /cureus. 4470 
Conclusion: DES is an effective, economical, and relatively safe drug in patients with CRPC.

Categories: Urology, Oncology

Keywords: castrate resistant prostate cancer, diethylstilbestrol

\section{Introduction}

Prostate cancer is a hormone-responsive disease. Androgen ablation is used as a primary treatment in symptomatic metastatic disease and as an adjuvant to radiation treatment in localized moderate- to high-risk cases [1]. Androgen deprivation therapy is achieved either by luteinizing hormone-releasing hormone (LHRH) analogs or bilateral orchiectomy.

Approximately $90 \%$ of patients respond well to the first line of hormonal treatment for a median time of 18-24 months [2]. In due course, however, most of these patients develop progressive disease even after the castrate levels of testosterone are achieved. This becomes evident by the progressive rise in serum prostate-specific antigen (PSA) levels leading to increased numbers of bony metastatic lesions seen via bone scan. At this time, the patient develops castration-resistant prostate cancer (CRPC), and few treatment options are available. CRPC can be treated with systemic chemotherapy with compounds such as docetaxel and cabazitaxel which carries a definite survival benefit [3-4]. However, chemotherapy is accompanied by its inherent complications, and patients' tolerance becomes the limiting factor.

Further hormonal manipulation in CRPC patients can be achieved via agents including estrogens, abiraterone, and enzalutamide [5]. An overall survival benefit is achieved with the latter two agents, but still, 10\%-20\% of patients are refractory to this form of treatment [6]. Additionally, a considerable cost of these medications has limited their wider use in lowermiddle-income countries like Pakistan.

Diethylstilbestrol (DES) is a synthetic estrogen that has shown efficacy in the treatment of prostate cancer dating back to the 1940s [7]. Orchiectomy and DES were used as first-line treatments for the next two decades until studies by the Veterans Administration Co-operative Urological Research Group (VCURG), reported significant thromboembolic events caused by DES at doses of $5 \mathrm{mg}$ daily [8-9]. The development and use of the LHRH analogs further decreased the use of DES in the treatment of prostate cancer because LHRH offered similar efficacy to that of orchiectomy with less physical and psychological trauma, and LHRH analogs did not cause increased thromboembolic complications as was seen with DES. Subsequently, the VCURG II study and some other smaller studies suggest that low doses of DES showed similar clinical efficacy as the higher dose with fewer cardiovascular side effects [10-12]. DES with thromboembolic prophylaxis has proven to be a less expensive alternative to the new costly hormonal agents, forcing clinicians to reconsider DES use in the management of CRPC. With its low cost and proven efficacy, we used it as a second-line treatment in combination with anticoagulant agents in patients with prostate cancer who developed resistance to complete androgen blockade (CAB).

\section{Materials And Methods}

From January 2011 to December 2016, all medical records of patients with a diagnosis of prostate cancer presenting at Shaukat Khanum Memorial Cancer Hospital and Research Centre, Lahore, were retrospectively reviewed. The patients with metastatic prostate cancer receiving castration either surgically by bilateral orchiectomy or medically by LHRH analogs were identified. This group of patients was followed clinically and with PSA levels at three monthly intervals, and those patients developing biochemical failure (i.e., rising PSA levels) were offered bicalutamide in a dose of 50-100 mg to achieve CAB. The cohort of patients showing a further rise in PSA after CAB were diagnosed with CRPC and selected for our study. This was further 
confirmed by serum testosterone levels below the castrate level of $50 \mathrm{ng} / \mathrm{dL}$. These patients were treated with $2.5 \mathrm{mg}$ DES initially, with some progressing to $5 \mathrm{mg}$ once daily. All patients were also prescribed aspirin $(75 \mathrm{mg})$ once daily for anticoagulation. Patients with a lack of complete follow-up information were excluded from the study. Patients who were on LHRH analog therapy remained on this treatment to maintain the castrate level of testosterone. These patients were further followed-up at three monthly intervals (or sooner if clinically indicated) to assess PSA levels. Radiological investigations like CT scan and bone scan were performed when indicated. The PSA response to treatment, time to disease progression, and adverse events were recorded and analyzed by IBM SPSS Statistics for Windows, Version 20.0. (IBM Corp., Armonk, NY).

Prostate-specific antigen before the start of DES therapy was taken as a baseline, and the lowest PSA value achieved after initiation of DES was labeled as the nadir value. The PSA response was quantified as good when $\mathrm{a} \geqslant 50 \%$ PSA decrease from baseline was achieved and considered as partial when the PSA response was $<50 \%$ from baseline. PSA progression was defined as $>50 \%$ PSA increase from the nadir values.

\section{Results}

A total of 91 patients were included in the study. The mean age was $66 \pm 8$ years, and the median baseline PSA at time of diagnosis was $150 \mathrm{ng} / \mathrm{mL}$ (range: $56-626 \mathrm{ng} / \mathrm{mL}$ ). Most of the patients were diagnosed with prostate cancer via transurethral ultrasound-guided biopsy and transurethral resection of the prostate (i.e., 36 [39.6\%] in each group). The median Gleason's score was eight. Eighty-two patients (90\%) had metastatic disease at the time of diagnosis. Hormonal ablation was achieved by LHRH analog in 20 patients (22.0\%) and bilateral orchiectomy in 64 patients (70.3\%). Seven of the patients (7.7\%), were initially provided LHRH analog and later underwent orchiectomy. The median decrease in PSA on androgen deprivation therapy (ADT) was -97.91\% (range: -99.9\%-30.88\%). The time needed for PSA progression was from 6.34 to 68.94 months with a median of 19.62 months. The patients' characteristics at the time of diagnosis are shown in Table 1. 


\section{Cureus}

\section{Characteristic}

Patient age (years; mean \pm SD)

Mode of diagnosis

TVP

TURP

TRUS Biopsy

Biopsy of distant metastases

Bone biopsy

Para-aortic lymph node biopsy

Bone marrow biopsy

Pelvic mass biopsy

Gleason's score distribution

GS 6

GS 7

GS 8-10

Distant metastases at diagnosis

Yes

No

PSA at diagnosis, median (range)
Patient data $(\mathrm{N}=91)$

$66 \pm 8$

n (\%)

$13(14.3 \%)$

$36(39.6 \%)$

$36(39.6 \%$

n (\%)

$2(2.1 \%)$

$2(2.1 \%)$

$1(1.1 \%)$

$1(1.1 \%)$

n (\%)

$3(3.3 \%)$

$31(34.1 \%)$

$55(60.4 \%)$

n (\%)

$82(90.1 \%)$

$9(9.9 \%)$

$150(56-626)$

\section{TABLE 1: Patients' characteristics.}

SD, standard deviation; TVP, transvesical prostatectomy; TURP, transurethral resection of the prostate; TRUS, transurethral ultrasound; GS, Gleason's score; PSA, prostate-specific antigen.

The response to ADT is shown in Table 2. 


\section{Cureus}

\section{Primary hormonal treatment}

LHRH analog

Bilateral orchiectomy

LHRH followed by orchiectomy

Change in PSA\%, median (range)

PFD months, median (range) n (\%)

$20(22.0 \%)$

$64(70.3 \%)$

$7(7.7 \%)$

$-97.91(-99.9,-30.88)$

$19.62(6.34,68.94)$

\section{TABLE 2: Types and response to first-line hormonal treatment.}

LHRH, luteinizing hormone-releasing hormone; PSA, prostate-specific antigen; PFD, progression free duration.

The patients' response to DES therapy is shown in Table 3.

\section{Therapy}

PSA at the start of therapy

Follow-up months

Overall PSA response to DES

Yes

No

Change in PSA\%, median (range)

Overall PFD, median (range)

PSA response to DES

$\geq 50 \%$ PSA response

$<50 \%$ PSA response

No response

\section{Median (range)}

$68.45 \mathrm{ng} / \mathrm{mL}(4.48-1,639 \mathrm{ng} / \mathrm{mL})$

$13.6(6.5-22)$

n (\%)

$78(85.7 \%)$

$13(14.3 \%)$

$-55.52(-99.9,+422)($

6.98 months $(1.77-34.38)$

n (\%) Median time to progression

$56(61.5 \%) \quad 8.96$ months

$22(24.2 \%) \quad 3.5$ months

$13(14.3 \%) \quad$ NA

\section{TABLE 3: PSA response to DES therapy.}

PSA, prostate-specific antigen; DES, diethylstilbestrol; PFD, progression free duration; NA, not applicable.

The variations in PSA response to DES therapy is shown in Figure 1. 


\section{Cureus}

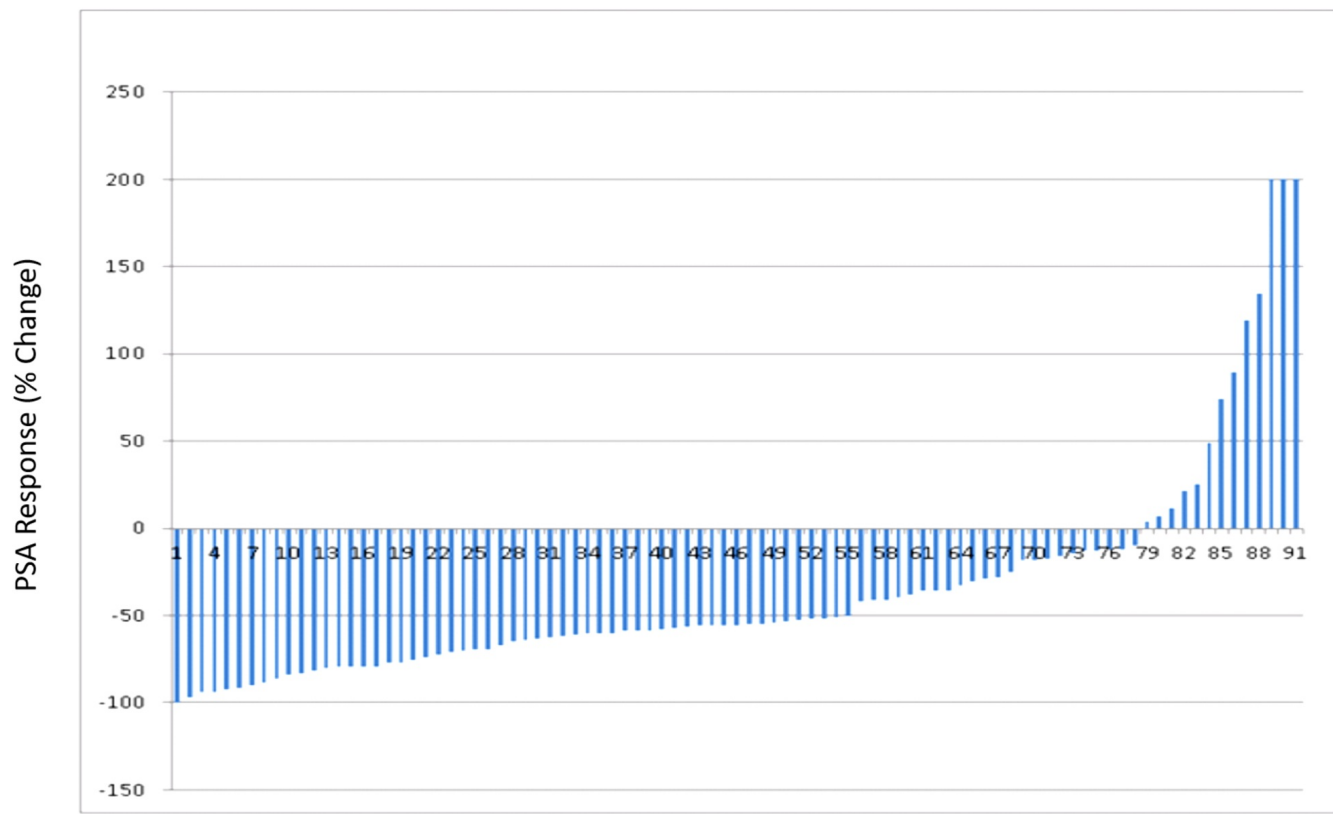

Total Number of Patients (91)

FIGURE 1: Water fall plot showing variations in PSA response to DES therapy.

PSA, prostate-specific antigen; DES, diethylstilbestrol.

Further treatments for patients in whom DES therapy failed are presented in Table 4.

\section{Treatment}

Palliative chemotherapy

Abiraterone

Ketoconazole

Palliative care n (\%)

$26(28.6 \%)$

$4(4.4 \%)$

$1(1.1 \%)$

$31(34.1 \%)$

\section{TABLE 4: Further treatment after DES failure.}

DES, diethylstilbestrol.

The detail of patients suffering from complications after using DES is shown in Table 5 . 


\section{Cureus}

\begin{tabular}{ll} 
DES adverse effects & $\mathbf{n}(\%)$ \\
\hline Deep venous thrombosis & $2(2.2 \%)$ \\
Myocardial infarction & $4(4.4 \%)$ \\
Pulmonary embolism & $1(1.1 \%)$ \\
Cerebrovascular accident & $1(1.1 \%)$ \\
Liver toxicity & $2(2.2 \%)$ \\
Gynecomastia & $11(12.08 \%)$
\end{tabular}

\section{TABLE 5: Adverse effects of DES therapy.}

DES, diethylstilbestrol.

Patients suffering from gynecomastia were managed with irradiation in nine patients; two required subcutaneous mastectomies for symptomatic relief. Two patients suffered from liver toxicity and DES had to be stopped.

\section{Discussion}

Diethylstilbestrol is a synthetic estrogen first manufactured in 1938. Its exact mechanism of action in CRPC is not clear, but it appears to be multifactorial. DES reduces serum testosterone levels by suppressing the hypothalamic testicular axis [13]. It also induces changes in adrenal androgen, dehydroandrostenedione (DHEA), and its sulfate derivative (DHEAS). It demonstrates a direct apoptotic effect on prostate cancer cells and inhibits DNA synthesis and angiogenesis [14-15].

In our study, DES appears to have significant activity in CRPC patients as 78\% of patients showed an overall PSA response to it. A PSA response of $\geqslant 50 \%$ was achieved in 56 patients (61.5\%), a rate similar to a previously published series in which DES was used as second-line hormonal therapy showing a response rate of $43 \%$ and $63 \%$ of patients [16-17]. In our series, it was also observed that patients who showed a PSA response $\geqslant 50 \%$ had a longer duration of stable disease as compared to patients who had a $<50 \%$ PSA response. This finding was also substantiated in studies by other authors. Shamash et al. observed that PSA decline $>50 \%$ one month after commencement of DES and dexamethasone therapy was found to predict a favorable prognosis with a median time to PSA progression more than one year and median survival of more than one year [18].

The major limitation in the use of DES for patients with CRPC is its availability. The fact that this medication is manufactured by selected pharmaceutical companies in a fixed dose formulation limits the prescription options. In our series, the drug was prescribed in a dose of 2.5-5 mg only. Because DES is available in 5-mg tablets, we could halve the tablets to create the 2.5-mg dose. Even with this relatively high dose administration, the drug tolerance profile was reasonable in most of our patients. Only eight (8.7\%) patients experienced thromboembolic events. In earlier studies, anticoagulants were not used routinely. Chang et al. reported a higher incidence of cardiovascular events in the DES group compared to the flutamide group (33.3\% vs. $17.6 \%$, respectively) [19]. Regular use of anticoagulants with DES significantly reduces 
cardiovascular complications [20]. Bosset et al. reported a 5\% incidence rate of thromboembolic event in patients taking DES with anticoagulants [21]. Many studies confirmed the use of anticoagulants with DES reduces thromboembolic events, but no particular anticoagulant has been recommended. Comparing warfarin with aspirin, Oh et al. reported a $9 \%$ thromboembolic event with daily 2-mg warfarin use, which was similar to that seen with aspirin [22].

Gynecomastia, although not bothersome, was reported in 11 of our patients. This can be prevented by prophylactic irradiation to the breast at a dose of 4-6 Gy either as a single or divided doses. Established gynecomastia is difficult to treat but may be managed with external irradiation to prevent further progression or by subcutaneous mastectomy [23-24]. Liver toxicity with deranged liver function test (LFT) results was seen in two patients. On cessation of DES, the LFTs results returned to normal.

The present study has several limitations. The data were retrospectively retrieved, and the study cohort was small. Imaging assessment to measure the response or progression was not routinely performed. Despite these limitations, the study revealed a significant response to DES in patients with CRPC.

As mentioned earlier, newer agents including abiraterone and enzalutamide have been developed noting the persistent activity of the androgen receptors in CRPC. The high costs become a major limitation to their use in patients in our part of the world. Because of its lower cost and considerable efficacy, DES is an effective alternative to these newer agents, despite its known adverse effects, until the newer treatments become available at more affordable costs.

\section{Conclusions}

Diethylstilbestrol is an effective treatment option in patients with CRPC for a modest duration and is safer when used with aspirin. It is a logical choice in lower middle-economy countries like Pakistan, where the more expensive newer hormonal therapies are financially inaccessible for a major part of the population.

\section{Additional Information \\ Disclosures}

Human subjects: Consent was obtained by all participants in this study. Institutional Review Board (IRB) of Shaukat Khanum Memorial Cancer hospital and Research Center, Lahore, Pakistan issued approval EXMPT-10-01-18-01. . IRB considers the fact that all the information to be collected in this study is already existing in medical records. . IRB further considers that this information will be recorded on the data extraction sheet in such a manner that subjects cannot be identified directly through use of codes. On the basis above, IRB grants you an exempt status. Animal subjects: All authors have confirmed that this study did not involve animal subjects or tissue. Conflicts of interest: In compliance with the ICMJE uniform disclosure form, all authors declare the following: Payment/services info: All authors have declared that no financial support was received from any organization for the submitted work.

Financial relationships: All authors have declared that they have no financial relationships at present or within the previous three years with any organizations that might have an interest in the submitted work. Other relationships: All authors have declared that there are no other relationships or activities that could appear to have influenced the submitted work.

\section{References}

1. Pagliarulo V, Bracarda S, Eisenberger MA, Mottet N, Schröder FH, Sternberg CN, Studer UE: Contemporary role of androgen deprivation therapy for prostate cancer . Eur Urol. 2012, 61:11-25. 10.1016/j.eururo.2011.08.026 
2. Scher HI, Halalbi S, Tannock I, et al.: Design and end points of clinical trials for patients with progressive prostate cancer and castrate levels of testosterone: recommendations of the Prostate Cancer Clinical Trials Working Group. J Clin Oncol. 2008, 26:1148-1159. 10.1200/JCO.2007.12.4487

3. Tannock IF, De Wit R, Berry WR, et al.: Docetaxel plus prednisolone or mitoxantrone plus prednisolone for advanced prostate cancer. New Engl J Med. 2004, 351:1502-1512. 10.1056/NEJMoa040720

4. De Bono JS, Oudard S, Ozguroglu M, et al.: Prednisone plus cabazitaxel or mitoxantrone for metastatic castration-resistant prostate cancer progressing after docetaxel treatment: a randomised open-label trial. Lancet. 2010, 376:1147-1154. 10.1016/S0140-6736(10)61389-X

5. De Bono JS, Logothetis CJ, Molina A, et al.: Abiraterone and increased survival in metastatic prostate cancer. N Engl J Med. 2011, 364:1995-2005. 10.1056/NEJMoa1014618

6. Ryan CJ, Smith MR, Fizazi K, et al.: 7530 final overall survival (OS) analysis of COU-AA-302, a randomized phase 3 study of abiraterone acetate (AA) in metastatic castration-resistant prostate cancer (MCRPC) patients (PTS) without prior chemotherapy. Ann Oncol. 2014, 25:255-279. 10.1093/annonc/mdu336.1

7. Huggins C, Hodges CV: The effect of castration, of estrogen and of androgen injection on serum phosphatases in metastatic carcinoma of the prostate. CA Cancer J Clin. 1972, 22:232240. 10.3322/canjclin.22.4.232

8. Veterans Administration Co-operative Urological Research Group: Treatment and survival of patients with cancer of the prostate. Surg Gynecol Obstet. 1967, 124:1011-1017.

9. Veterans Administration Cooperative Urological Research Group: Carcinoma of the prostate: treatment comparisons. J Urol. 1967, 98:516-522. 10.1016/S0022-5347(17)62926-4

10. Blackard CE, Doe RP, Mellinger GT, Byar DP: Incidence of cardiovascular disease and death in patients receiving diethylstilbestrol for carcinoma of the prostate. Cancer. 1970, 26:249-256. 10.1002/1097-0142(197008)26:2<249::AID-CNCR2820260202>3.0.CO;2-7

11. Byar DP, Corle DK: Hormone therapy for prostate cancer: results of the Veterans Administration Cooperative Urological Research Group studies. NCI Monogr. 1988, 46:165170.

12. Smith DC, Redman BG, Flaherty LE, Li L, Strawderman M, Pienta KJ: A phase II trial of oral diethylstilbestrol as a second line hormonal agents in advanced prostate cancer. Urology. 1998, 52:257-260. 10.1016/S0090-4295(98)00173-3

13. Kitahara S, Yoshida K, Ishizaka K, Kageyama Y, Kawakami S, Tsujii T, Oshima H: Stronger suppression of serum testosterone and FSH levels by a synthetic estrogen than by castration or an LH-RH agonist. Endocr J. 1997, 44:527-532. 10.1507/endocrj.44.527

14. Qiu CH, Ohe M, Koibuchi N, Matsuzaki S: Apoptosis in the epididymal epithelium of adult male golden hamster exposed to diethylstilbestrol. J Histochem Cytochem. 2004, 52:187-192. 10.1177/002215540405200206

15. Ho SM: Estrogens and anti-estrogens: key mediators of prostate carcinogenesis and new therapeutic candidates. J Cell Biochem. 2004, 91:491-503. 10.1002/jcb.10759

16. Manikandan R, Srirangam SJ, Pearson E, Brown SC, O’Reilly P, Collins GN: Diethylstilbestrol versus bicalutamide in hormone refractory prostate cancer: a prospective randomized trial. Urol Int. 2005, 75:217-221. 10.1159/000087797

17. Grenader T, Plotkin Y, Gips M, Cherny N, Gabizon A: Diethylstilbestrol for the treatment of patients with castration-resistant prostate cancer: retrospective analysis of a single institution experience. Oncol Rep. 2014, 31:428-434. 10.3892/or.2013.2852

18. Shamash J, Stebbing J, Sweeney C, et al.: A validated prognostic index predicting response to dexamethasone and diethylstilbestrol in castrate-resistant prostate cancer. Cancer. 2010, 116:3595-3602. 10.1002/cncr.25194

19. Chang A, Yeap B, Davis T, et al.: Double-blind, randomized study of primary hormonal treatment of stage D2 prostate carcinoma: flutamide versus diethylstilbestrol. J Clin Oncol. 1996, 14:2250-2257. 10.1200/JCO.1996.14.8.2250

20. Shamash J, Powles T, Sarker SJ, et al.: A multi-centre randomised phase III trial of dexamethasone vs dexamethasone and diethylstilbestrol in castration resistant prostate cancer: immediate vs deferred diethylstilbestrol. Br J Cancer. 2011, 104:620-628. 10.1038/bjc.2011.7

21. Bosset PO, Albiges L, Seisen T, de la Motte Rouge T, Phé V, Bitker MO, Rouprêt M: Current role of diethylstilbestrol in the management of advanced prostate cancer. BJU Int. 2012, 


\section{Cureus}

110:826-829. 10.1111/j.1464-410X.2012.11206.x

22. Oh WK, Kantoff PW, Weinberg V, et al.: Prospective, multicenter, randomized phase II trial of the herbal supplement, PC-SPES, and diethylstilbestrol in patients with androgen-

independent prostate cancer. J Clin Oncol. 2004, 22:3705-3712. 10.1200/JCO.2004.10.195

23. Malkowicz SB: The role of diethylstilbestrol in the treatment of prostate cancer . Urology. 2001, 58:108-113. 10.1016/S0090-4295(01)01252-3

24. Dobs A, Darkes MJ: Incidence and management of gynecomastia in men treated for prostate cancer. J Urol. 2005, 174:1737-1742. 10.1097/01.ju.0000176461.75794.f8 